\title{
63. On Equations Defining Abelian Varieties and Modular Functions
}

\author{
By Shoji KoIzUmi*) \\ Universität Bonn and University of Tsukuba \\ (Communicated by Kunihiko KodaIRA, M. J. A., Oct. 12, 1978)
}

To a pair $(z, e)$ of a point $z$ of the Siegel upper half space $\mathcal{H}_{n}$ of degree $n$ and an $n$-square matrix $e$ with coefficients in $Z$ and $\operatorname{det} e \neq 0$, we can attach a complex torus $C /\langle z, e\rangle$ with the period lattice subgroup $\langle z, e\rangle$ of $C^{n}$ generated by the column vectors of $(z, e)$. For a vector $k=\left(\begin{array}{l}k^{\prime} \\ k^{\prime \prime}\end{array}\right) \in \boldsymbol{R}^{2 n}$, the theta function $\vartheta[k](z \mid x)$ is defined by

$$
\vartheta[k](z \mid x)=\sum_{r \in \mathbb{Z}^{n}} e\left(\frac{1}{2} t\left(r+k^{\prime}\right) z\left(r+k^{\prime}\right)+{ }^{t}\left(r+k^{\prime}\right)\left(x+k^{\prime \prime}\right)\right),
$$

which is a holomorphic function of $(z, x) \in \mathcal{H}_{n} \times \boldsymbol{C}^{n}$. Given $\beta \in \boldsymbol{Z}_{+}$, $U\left(\beta^{t} e\right)$ denotes a complete set of representatives of $\beta^{-1 t} e^{-1} Z^{n} \bmod Z^{n}$. A map $\varphi^{(z)}$ from $C^{n}$ to the projective space $P \beta^{n \mid \text { det el-1-1}}(C)$ defined by $x \mapsto\left(\cdots, \vartheta\left[\begin{array}{c}k^{\prime} \\ 0\end{array}\right](z \mid x), \cdots\right)_{k^{\prime} \in U\left(\beta \beta^{t}\right)}$ induces a projective embedding of $C^{n} /\langle z, e\rangle$ if $\beta \geqslant 3$. The $\operatorname{Im}\left(\varphi^{(z)}\right)$ is an abelian variety, which is denoted by $A(z)$. The purpose of this note is to write down explicitly a system of equations defining $A(z)$ and to show that the set of quotients of coefficients of the equations generates the field of modular functions with respect to the principal congruence subgroup $\Gamma_{t_{e}}(\beta)$.

We shall indicate some definitions and notations. For a commutative ring $R$ having the unity $1, \boldsymbol{M}(n \times \alpha, R)$ (or $\boldsymbol{M}(n, R)$, resp.) is the set of $(n \times \alpha)$-matrices (or $n$-square matrices) with coefficients in $R$; in particular, $M(n \times 1, R)$ is denoted by $R^{n}$. For a matrix $e \in M(n, Z)$ with det $e \neq 0$, the paramodular group $\Gamma_{t_{e}}$ or the principal congruence subgroup $\Gamma_{t_{e}}(\beta)$ of level $\beta, \beta \in Z_{+}$, is defined, respectively, by $\Gamma_{t_{e}}$ $=\left\{\left.M \in \boldsymbol{M}(2 n, \boldsymbol{Z})\right|^{t} \boldsymbol{M}\left(\begin{array}{cc}0 & -{ }^{t} e \\ e & 0\end{array}\right) \boldsymbol{M}=\left(\begin{array}{cc}0 & -{ }^{t} e \\ e & 0\end{array}\right)\right\} \quad$ or $\quad \Gamma_{t_{e}}(\beta)=\left\{M \in \Gamma_{t_{e}} \mid M\right.$ $\left.=1_{2 n}+M^{\prime}\left(\begin{array}{cc}e & 0 \\ 0 & { }^{t} e\end{array}\right), M^{\prime} \in M(2 n, Z)\right\}$. When we write $k=\left(\begin{array}{l}k^{\prime} \\ k^{\prime \prime}\end{array}\right) \in \boldsymbol{R}^{2 n}, k^{\prime}$ are $k^{\prime \prime}$ are upper and lower halves of $k$ in $\boldsymbol{R}^{n}$. For $e$ (or $\beta$ ) as above, $U(e)$ (or $U(\beta)$ ) is a complete set of representatives of $e^{-1} Z^{n}$ (or $\beta^{-1} Z^{n}$ ) modulo $\boldsymbol{Z}^{n}$. On the other hand, the residue group $e^{-1} \boldsymbol{Z}^{n} / \boldsymbol{Z}^{n}$ and its character group are denoted, respectively, by $\tilde{U}(e)$ and $\tilde{U}^{*}(e)$. We put $\boldsymbol{e}(t)=\exp (2 \pi \sqrt{-1} t)$ for $t \in \boldsymbol{C}$.

*) Supported in part by the S.F.B. of Universität Bonn. 
More precise details and proofs will be discussed in a separate paper.

1. Theta relations of higher degree. Let $\alpha$ be an integer $\geqslant 2$ and let $T$ be a matrix in $M(\alpha, Z)$ defined by

$$
T=\left(\begin{array}{ccccrr}
1 & \alpha-1 & 0 & \cdots & 0 & 0 \\
1 & -1 & \alpha-2 & \cdots & 0 & 0 \\
1 & -1 & -1 & \cdots & 0 & 0 \\
\vdots & \vdots & \vdots & \cdot & \vdots & \vdots \\
1 & -1 & -1 & \cdots & -1 & 0 \\
1 & -1 & -1 & \cdots & -1 & -1
\end{array}\right)
$$

If we write ${ }^{t} T^{-1}=T^{*}$, we have

$$
T^{*}={ }^{t} T^{-1}=\left(\begin{array}{cccccc}
\alpha^{-1} & \alpha^{-1} & 0 & \cdots & 0 & 0 \\
\alpha^{-1} & -\alpha^{-1}(\alpha-1)^{-1} & (\alpha-1)^{-1} & \cdots & 0 & 0 \\
\alpha^{-1} & -\alpha^{-1}(\alpha-1)^{-1} & -(\alpha-1)^{-1}(\alpha-2)^{-1} & \cdots & 0 & 0 \\
\vdots & \vdots & \vdots & \cdot & \vdots & \vdots \\
\alpha^{-1} & -\alpha^{-1}(\alpha-1)^{-1} & -(\alpha-1)^{-1}(\alpha-2)^{-1} & \cdots & -6^{-1} & 2^{-1} \\
\alpha^{-1} & -\alpha^{-1}(\alpha-1)^{-1} & -(\alpha-1)^{-1}(\alpha-2)^{-1} & \cdots & -6^{-1} & -2^{-1}
\end{array}\right) .
$$

For $\alpha-1$ vectors $l_{i}=\left(\begin{array}{l}l_{i}^{\prime} \\ l_{i}^{\prime \prime}\end{array}\right) \in \boldsymbol{R}^{2 n}$, a holomorphic function of $\left(z, y_{1}\right.$,

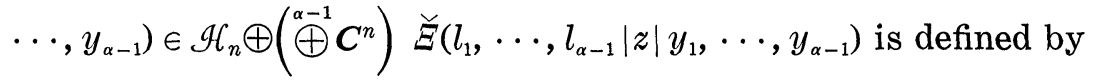

$$
\breve{\Xi}\left(l_{1}, \cdots, l_{\alpha-1}|z| y_{1}, \cdots, y_{\alpha-1}\right)=\sum_{\substack{p_{i} \in U(i) \\
i=\alpha-1, \cdots, 2}} \vartheta\left[\begin{array}{c}
l_{1}^{\prime}-p_{\alpha-1} \\
l_{1}^{\prime \prime}
\end{array}\right]\left(\alpha(\alpha-1) z \mid y_{1}\right)
$$

$$
\begin{aligned}
& \times \vartheta\left[\begin{array}{c}
l_{2}^{\prime}+p_{\alpha-1}-p_{\alpha-2} \\
l_{2}^{\prime \prime}
\end{array}\right]\left((\alpha-1)(\alpha-2) z \mid y_{2}\right) \cdots \vartheta\left[\begin{array}{c}
l_{\alpha-2}^{\prime}-p_{3}+p_{2} \\
l_{\alpha-2}^{\prime \prime}
\end{array}\right]\left(6 z \mid y_{\alpha-2}\right) \\
& \times \vartheta\left[\begin{array}{c}
l_{\alpha-1}^{\prime}+p_{2} \\
l_{\alpha-1}^{\prime \prime}
\end{array}\right]\left(2 z \mid y_{\alpha-1}\right) .
\end{aligned}
$$

Then we have (Appendix in [2])

$$
\begin{aligned}
\prod_{i=1}^{\alpha} \vartheta\left[\begin{array}{c}
k_{i}^{\prime} \\
k_{i}^{\prime \prime}
\end{array}\right]\left(z \mid x_{i}\right) \\
\quad=\sum_{p \in U(\alpha)} \vartheta\left[\begin{array}{c}
l_{0}^{\prime}+p \\
l_{0}^{\prime \prime}
\end{array}\right](\alpha z \mid y) \check{\Xi}\left(l_{1}+\left(\begin{array}{c}
p \\
0
\end{array}\right), l_{2}, \cdots, l_{\alpha-1}|z| y_{1}, \cdots, y_{\alpha-1}\right),
\end{aligned}
$$

where $\quad\left(l_{0} l_{1} \cdots l_{\alpha-1}\right)=\left(\begin{array}{l}l_{0}^{\prime} l_{1}^{\prime} \cdots l_{\alpha-1}^{\prime} \\ l_{0}^{\prime \prime} l_{1}^{\prime \prime} \cdots l_{\alpha-1}^{\prime \prime}\end{array}\right)=\left(\begin{array}{c}\left(k_{1}^{\prime} k_{2}^{\prime} \cdots k_{\alpha}^{\prime}\right) T^{*} \\ \left(k_{1}^{\prime \prime} k_{2}^{\prime \prime} \cdots k_{\alpha}^{\prime \prime}\right) T\end{array}\right)$ and $\left(y y_{1} \cdots y_{\alpha-1}\right)$ $=\left(x_{1} x_{2} \cdots x_{\alpha}\right) T$.

Let $\chi$ be a character in $\tilde{U}^{*}(\alpha)$. We put

$$
\begin{aligned}
& \breve{\Xi}\left(\chi\left|l_{1}, \cdots, l_{\alpha-1}\right| z \mid y_{1}, \cdots, y_{\alpha-1}\right) \\
& \quad=\sum_{p \in U(\alpha)} \chi(-p) \breve{\Xi}\left(l_{1}+\left(\begin{array}{c}
p \\
0
\end{array}\right), l_{2}, \cdots, l_{\alpha-1}|z| y_{1}, \cdots, y_{\alpha-1}\right) .
\end{aligned}
$$


Then, under the same notations as in (1.3) we have

$$
\begin{aligned}
& \sum_{p \in U(\alpha)} \chi(p) \prod_{i=1}^{\alpha} \vartheta\left[k_{i}+\left(\begin{array}{l}
p \\
0
\end{array}\right)\right]\left(z \mid x_{i}\right) \\
& =\left(\sum_{p \in U(\alpha)} \chi(p) \vartheta\left[l_{0}+\left(\begin{array}{c}
p \\
0
\end{array}\right)\right](\alpha z \mid y)\right) \breve{\Xi}\left(\chi\left|l_{1}, \cdots, l_{\alpha-1}\right| z \mid y_{1}, \cdots, y_{\alpha-1}\right) \text {. }
\end{aligned}
$$

Let $\left(k_{01}, k_{02}, \cdots, k_{0 \alpha}\right)$ and $\left(k_{11}, k_{12}, \cdots, k_{1 \alpha}\right)$ be two systems of vectors in $R^{2 n}$. As in (1.3), we put

and

$$
\left(l_{j 0} l_{j 1} \cdots l_{j(\alpha-1)}\right)=\left(\begin{array}{l}
l_{j 0}^{\prime} l_{j 1}^{\prime} \cdots l_{j(\alpha-1)}^{\prime} \\
l_{j 0}^{\prime \prime} l_{j 1}^{\prime \prime} \cdots l_{j(\alpha-1)}^{\prime \prime}
\end{array}\right)=\left(\begin{array}{l}
\left(k_{j 0}^{\prime} k_{j 1}^{\prime} \cdots k_{j \alpha}^{\prime}\right) T^{*} \\
\left.k_{j 0}^{\prime \prime} k_{j 1}^{\prime \prime} \cdots k_{j \alpha}^{\prime \prime}\right) T
\end{array}\right), \quad(j=0,1),
$$

$$
\left(y y_{1} \cdots y_{\alpha-1}\right)=\left(x_{1} x_{2} \cdots x_{\alpha}\right) T \text {. }
$$

If $l_{00}^{\prime} \equiv l_{10}^{\prime}\left(\bmod \alpha^{-1} Z^{n}\right)$ and $l_{00}^{\prime \prime}=l_{10}^{\prime \prime}$, then we have

$$
\begin{aligned}
\breve{\Xi}\left(\chi \mid l_{11},\right. & \left.\cdots, l_{1(\alpha-1)}|z| y_{1}, \cdots, y_{\alpha-1}\right)\left(\sum_{p \in U(\alpha)} \chi(p) \prod_{i=1}^{\alpha} \vartheta\left[k_{0 i}+\left(\begin{array}{c}
p \\
0
\end{array}\right)\right]\left(z \mid x_{i}\right)\right. \\
= & \chi\left(l_{10}^{\prime}-l_{00}^{\prime}\right) \check{\Xi}\left(\chi\left|l_{01}, \cdots, l_{0(\alpha-1)}\right| z \mid y_{1}, \cdots, y_{\alpha-1}\right) \\
& \times\left(\sum_{p \in U(\alpha)} \chi(p) \prod_{i=1}^{\alpha} \vartheta\left[k_{1 i}+\left(\begin{array}{c}
p \\
0
\end{array}\right)\right]\left(z \mid x_{i}\right) .\right.
\end{aligned}
$$

In particular we consider this formula (1.6) in the case where $k_{j 1}^{\prime \prime}=k_{j 2}^{\prime \prime}=\cdots=k_{j \alpha}^{\prime \prime}=0, j=0,1$, and $x_{1}=x_{2}=\cdots=x_{\alpha}=x$. Under this assumption, we have $l_{j 0}^{\prime \prime}=\cdots=l_{j(\alpha-1)}^{\prime \prime}=0$ and $y_{1}=\cdots=y_{\alpha-1}=0$. Thus we put

$$
\Xi\left(\chi\left|l_{1}^{\prime}, \cdots, l_{\alpha-1}^{\prime}\right| z\right)=\breve{\Xi}\left(\chi\left|\left(\begin{array}{c}
l_{1}^{\prime} \\
0
\end{array}\right), \cdots,\left(\begin{array}{c}
l_{\alpha-1}^{\prime} \\
0
\end{array}\right)\right| z \mid 0, \cdots, 0\right)
$$

for $\chi \in \tilde{U}^{*}(\alpha)$ and $\left(l_{1}^{\prime}, \cdots, l_{\alpha-1}^{\prime}\right) \in M(n \times(\alpha-1), \boldsymbol{R})$.

After suitable substitutions we have a formula containing

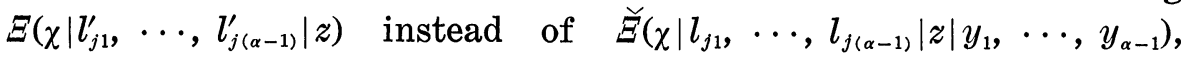
$(j=0,1)$, which is a special case of the formula (1.6).

2. Equations defining the abelian variety $A(z)$. Now, besides $e \in M(n, Z)$ with det $e \neq 0$ we fix two integers $\beta \geqslant 3$ and $\alpha>1$ such that $\beta$ is divisible by $\alpha$. We also fix a complete set $U\left(\beta^{t} e\right)$ of representatives once for all. Let $\left\{X\left(\tilde{k}^{\prime}\right) \mid k^{\prime} \in U\left(\beta^{t} e\right)\right\}$ be a set of independent indeterminates, bijectively corresponding to $\tilde{U}\left(\beta^{t} e\right)$, where $\tilde{k}^{\prime}$ is the congruence class determined by $k^{\prime} \in U\left(\beta^{t} e\right)$. $\left(\cdots, X\left(\tilde{k}^{\prime}\right), \cdots\right)$ can be considered as the coordinate variables of the ambient projective space of $A(z)$, which is the projective embedding of the complex torus $C^{n} \mid\langle z, e\rangle$ by $x \mapsto\left(\cdots, \vartheta\left[\begin{array}{c}k^{\prime} \\ 0\end{array}\right](\beta z \mid \beta x), \cdots\right)$.

For $\tilde{l}^{\prime}$ or $\left(\tilde{l}^{\prime}, \chi\right), \tilde{l}^{\prime} \in \tilde{U}\left(\beta^{t} e\right)$ and $\chi \in \tilde{U}^{*}(\alpha)$, a set $L\left(\tilde{l}^{\prime}\right)$ of indices or a set $F\left(\chi, \tilde{l}^{\prime}\right)$ of holomorphic functions on $\mathcal{H}_{n}$ are defined respectively by

$$
L\left(\tilde{l}^{\prime}\right)=\left\{\left(k_{1}^{\prime}, \cdots, k_{\alpha}^{\prime}\right) \mid k_{i}^{\prime} \in U\left(\beta^{t} e\right), i=1, \cdots, \alpha ; \sum_{i=1}^{\alpha} \tilde{k_{i}^{\prime}}=\tilde{l}^{\prime}\right\},
$$

and 


$$
\begin{aligned}
F\left(\chi, \tilde{l}^{\prime}\right)= & \left\{\boldsymbol{g}\left(\chi\left|l_{1}^{\prime}, \cdots, l_{\alpha-1}^{\prime}\right| \beta z\right) \mid\left(l_{1}^{\prime} \cdots l_{\alpha-1}^{\prime}\right)=\left(k_{1}^{\prime} \cdots k_{\alpha}^{\prime}\right) T_{1}^{*},\right. \\
& \left.\left(\tilde{k}_{1}^{\prime}, \cdots, \tilde{k}_{\alpha}^{\prime}\right) \in L\left(\tilde{l}^{\prime}\right)\right\},
\end{aligned}
$$

where $T_{1}^{*}$ is the $\alpha \times(\alpha-1)$-matrix obtained from $T^{*}$ by excluding the first column.

$\left(k_{j 1}^{\prime}, \cdots, k_{j \alpha}^{\prime}\right)$ and $\left(l_{j 0}^{\prime}, \cdots, l_{j(\alpha-1)}^{\prime}\right)$ being as in (1.6), for a triple $\left(\chi,\left(\tilde{k}_{0 i}^{\prime}\right)_{1 \leqslant i \leqslant \alpha},\left(\tilde{k}_{1 i}^{\prime}\right)_{1 \leqslant i \leqslant \alpha}\right)$ such that $\left(k_{0 i}^{\prime}\right)$ and $\left(k_{1 i}^{\prime}\right)$ belong to the same $L\left(\tilde{l}^{\prime}\right)$ for some $\tilde{l}^{\prime} \in \tilde{U}\left(\beta^{t} e\right)$, we define an $\alpha$-form ( $\alpha$-homogeneous polynomial) in variables $\{X(\tilde{k})\}_{\tilde{k} \in \tilde{U}\left(\beta t_{e}\right)}$ :

$$
\begin{aligned}
P\left(\chi\left|\left(\tilde{k_{0 i}^{\prime}}\right),\left(\tilde{k_{1 i}^{\prime}}\right)\right| X\left(\tilde{k^{\prime}}\right)\right)= & \Xi\left(\chi\left|l_{11}^{\prime}, \cdots, l_{1(\alpha-1)}^{\prime}\right| \beta z\right) \sum_{p \in U(\alpha)} \chi(p) \prod_{i=1}^{\alpha} X\left(\widetilde{k_{0 i}^{\prime}+p}\right) \\
& -\chi\left(l_{10}^{\prime}-l_{00}^{\prime}\right) \Xi\left(\chi\left|l_{01}^{\prime}, \cdots, l_{0((\alpha-1)}^{\prime}\right| \beta z\right) \\
\times & \sum_{p \in U(\alpha)} \chi(p) \prod_{i=1}^{\alpha} X\left(\widetilde{k_{1 i}^{\prime}+p}\right) .
\end{aligned}
$$

Furthermore, we define a set $I(z)$ of polynomials by

$$
\begin{aligned}
I(z)= & \left\{P\left(\chi \mid \tilde{k}_{0 i}^{\prime}\right),\left(\tilde{k}_{1 i}^{\prime}\right) \mid X\left(\tilde{k}^{\prime}\right)\right) \mid \chi \in \tilde{U}^{*}(\alpha),\left(k_{0 i}^{\prime}\right) \text { and }\left(k_{1 i}^{\prime}\right) \in L\left(\tilde{l}^{\prime}\right), \\
& \left.\tilde{l}^{\prime} \in \tilde{U}\left(\beta^{t} e\right)\right\}
\end{aligned}
$$

and for a point $z_{0} \in \mathcal{H}_{n}, I\left(z_{0}\right)$ is the set of $\alpha$-forms in $C\left[\left\{X\left(\tilde{\tilde{k}^{\prime}}\right)\right\}_{\tilde{k}^{\prime} \in \tilde{U}\left(\beta^{t} e\right)}\right]$ obtained from $I(z)$ through substituting $z$ by $z_{0}$.

Then our theorem is formulated in the following way:

Theorem. ( 0 ) If we substitute $\left(X\left(\tilde{k}^{\prime}\right)\right)$ by $\left(\vartheta\left[\begin{array}{c}k^{\prime} \\ 0\end{array}\right](\beta z \mid \beta x)\right)$ in $P\left(\chi\left|\left(\tilde{k}_{0 i}^{\prime}\right),\left(\tilde{k}_{1 i}^{\prime}\right)\right| X\left(\tilde{k}^{\prime}\right)\right)$, it vanishes identically as a function of $(z, x)$ $\in \mathcal{H}_{n} \times \boldsymbol{C}^{n}$.

( I ) For an arbitrary $\left(\chi, \tilde{l}^{\prime}, z_{0}\right) \in \tilde{U}^{*}(\alpha) \times \tilde{U}\left(\beta^{t} e\right) \times \mathcal{H}_{n}$, there is a function $\Xi\left(\chi\left|l_{1}^{\prime}, \cdots, l_{\alpha-1}^{\prime}\right| \beta z\right)$ in $F\left(\chi, \tilde{l}^{\prime}\right)$, which does not vanish at $z_{0}$.

(II) Given $z_{0} \in \mathcal{H}_{n}$, the space of $\alpha$-forms in $C\left[\left\{X\left(\tilde{k}^{\prime}\right)\right\}_{\tilde{k}^{\prime} \in \tilde{U}\left(\beta^{t} e\right)}\right]$ vanishing on $A\left(z_{0}\right)$ is spanned by $I\left(z_{0}\right)$.

(III) The abelian variety $A\left(z_{0}\right)$ is the common zero set of $I\left(z_{0}\right)$.

(IV) The quotient of two functions in an $F\left(\chi, \tilde{l}^{\prime}\right)$, whose denominator does not identically vanish, is a modular function with respect to $\Gamma_{t e}(\beta)$.

( V ) The field of modular functions with respect to $\Gamma_{t e}(\beta)$ is generated by

$$
\begin{aligned}
\bigcup_{\left(x, \tilde{l}^{\prime}\right) \in \tilde{U}^{*}(\alpha) \oplus U\left(\beta t_{e}\right)} & \left\{\Xi\left(\chi\left|l_{01}^{\prime}, \cdots, l_{0(\alpha-1)}^{\prime}\right| \beta z\right) / \Xi\left(\chi\left|l_{11}^{\prime}, \cdots, l_{1(\alpha-1)}^{\prime}\right| \beta z\right)\right. \\
& \mid \Xi\left(\chi\left|l_{01}^{\prime}, \cdots\right| \beta z\right) \text { and } \Xi\left(\chi\left|l_{11}^{\prime}, \cdots\right| \beta z\right) \in F\left(\chi, \tilde{l}^{\prime}\right), \\
& \left.\Xi\left(\chi\left|l_{11}^{\prime}, \cdots\right| \beta z\right) \neq 0\right\} .
\end{aligned}
$$

The assertions (0)-(III) are known in the case $\beta=4$ and $\alpha=2$ ([1], [3]). In the case $\beta=9$ and $\alpha=3$, the assertions (0)-(II) are proved in [5].

3. The Fourier expansion of $\Xi\left(\chi\left|l_{1}^{\prime}, \cdots, l_{\alpha-1}^{\prime}\right| z\right)$. We can determine series expansions of the functions $\breve{\Xi}\left(l_{1}, \cdots, l_{\alpha-1}|z| y_{1}, \cdots, y_{\alpha-1}\right)$ etc. explicitly, when $\left(l_{1}, \cdots, l_{\alpha-1}\right)$ and $\left(y_{1}, \cdots, y_{\alpha-1}\right)$ are given as in (1.3). Here we restrict ourselves to considering only the function $\Xi\left(\chi \mid l_{1}^{\prime}, \cdots\right.$, $\left.l_{\alpha-1}^{\prime} \mid z\right)$. 
Let $\alpha, \zeta, K$ and $O$ be, respectively, a prime number, a primitive $\alpha$-th root of the unity, the field $Q(\zeta)$ and the ring $Z[\zeta] . \operatorname{Tr} M$ means the trace of the matrix $M$, and on the other hand $\operatorname{tr}(\lambda), \lambda=\left(\begin{array}{c}\lambda_{(1)} \\ \vdots \\ \lambda_{(n)}\end{array}\right) \in K^{n}$, means the vector in $Q^{n}$ whose coefficients are given by the trace of the element $\lambda_{(i)}$ in $K$, over $\boldsymbol{Q}$.

Given $\chi \in \tilde{U}^{*}(\alpha)$ and $\lambda \in K^{n}$, we define a holomorphic function $\xi(\chi, \lambda, z)$ on $\mathcal{H}_{n}$ by

$$
\xi(\chi, \lambda, z)=\sum_{\sigma \in D^{n}} \chi\left(\alpha^{-1} \operatorname{tr} \sigma\right) e\left(\frac{1}{2} \operatorname{Tr}\left(\left(\operatorname{tr}\left((\lambda+\sigma)_{(i)}(\overline{\lambda+\sigma})_{(j)}\right)\right)_{(i, j)} z\right)\right),
$$

where $\left(\operatorname{tr}\left((\lambda+\sigma)_{(i)}(\overline{\lambda+\sigma})_{(j)}\right)\right)_{(i, j)}$ is a positive semi-definite symmetric matrix in $\boldsymbol{M}(n, \boldsymbol{Q})$.

For $\left(k_{1}^{\prime}, \cdots, k_{\alpha}^{\prime}\right) \in M(n \times \alpha, \boldsymbol{Q})$, if we put $\lambda=k_{1}^{\prime}+k_{2}^{\prime} \zeta+\cdots+k_{\alpha}^{\prime} \zeta^{\alpha-1}$ and $\left(l_{1}^{\prime}, \cdots, l_{\alpha-1}^{\prime}\right)$ as in (1.3), then we have

$$
\xi\left(\chi, \lambda, \alpha^{-1} z\right)=\Xi\left(\chi\left|l_{1}^{\prime}, \cdots, l_{\alpha-1}^{\prime}\right| z\right) \text {. }
$$

Using $\xi\left(\chi, \lambda, \frac{\beta}{\alpha} z\right)$ instead of $\Xi\left(\chi\left|l_{1}^{\prime}, \cdots, l_{\alpha-1}^{\prime}\right| \beta z\right)$ we can formulate Theorem in the previous section. In this case the set $F\left(\chi, \tilde{l}^{\prime}\right)$ in (2.2) is replaced by

$$
\mathscr{F}\left(\chi, \tilde{l}^{\prime}\right)=\left\{\xi\left(\chi, \lambda \frac{\beta}{\alpha} z\right) \mid \lambda \in \mathcal{U}\left(\beta^{t} e\right), \quad \operatorname{tr} \lambda^{\prime} \equiv-l^{\prime} \bmod \alpha \beta^{-1 t} e^{-1} Z^{n}\right\},
$$

where $U\left(\beta^{t} e\right)$ is a complete set of representatives of $\beta^{-1 t} e^{-1} \mathfrak{D}^{n} \bmod \mathfrak{D}^{n}$.

\section{References}

[1] J. Igusa: Theta functions. Die Grundlehren der Math. Wiss., bd. 194, Springer-Verlag, Berlin (1972).

[2] S. Koizumi: Theta relations and projective normality of abelian varieties. Amer. J. Math., 98, 865-889 (1976).

[ 3 ] D. Mumford: On the equations defining abelian varieties. I. Invent. Math., 1, 287-354 (1966).

[4] - : Varieties defined by quadratic equations. Questioni sulle Varieta Algebraiche, Corsi dal C.I.M.E., Edizioni Cremonese, Roma (1969).

[5] R. Sasaki: Analogs to Riemann relations in the case of degree three. Master thesis, Tokyo Univ. of Education (1974) (unpublished).

[6] T. Sekiguchi: On the cubics defining abelian varieties (to appear).

[7] C. L. Siegel: Moduln abelscher Funktionen. Gesammelte Abhandlungen, bd. III, Springer-Verlag, Berlin, pp. 373-435 (1966).

[ 8 ] - U Über Moduln abelscher Funktionen. Nachr. Akad. Wiss. in Göttigen. II, nr. 4, pp. 79-96 (1971). 